\title{
Estudio preliminar de la usabilidad de tres tamaños de teclados basados en Interfaces Cerebro-Computador por pacientes con ELA
}

\section{Medina-Juliá, M. Teresa; Fernández-Rodríguez, Álvaro; Velasco-Álvarez, Francisco y Ron-Angevin, Ricardo}

Departamento de Tecnología Electrónica, Universidad de Málaga, Málaga, España. maytemed@uma.es, afernandezrguez@uma.es, fvelasco@uma.es,rron@uma.es

\begin{abstract}
Brain-Computer Interface-based spellers which use the P300 bio-potential as control signal have been widely studied. Nevertheless, the optimal speller size has not been properly presented for motor-disabled people. In the present work tree different speller sizes were assessed through the usability approach - in terms of effectiveness, efficiency and satisfaction - to offer a proper one. Four patients diagnosed with amyotrophic lateral sclerosis evaluated the speller sizes. They achieved worse performance with the small size, especially in the effectiveness and satisfaction dimensions; while with the medium size they achieved better performance, highlighting satisfaction. The results show that the speller size must be considered while designing a new paradigm for motor-disabled.
\end{abstract}

Keywords: Amyotrophic Lateral Sclerosis (ALS), brain-computer interface (BCI), speller, P300, speller size, usability.

\begin{abstract}
Resumen
Con referencia a los teclados basados en Interfaces Cerebro Computador que utilizan el biopotencial P300 como señal de control, el tamaño óptimo para personas con discapacidad motora no se ha presentado propiamente. En este trabajo se evaluaron tres tamaños de teclados mediante el enfoque de usabilidad - en términos de efectividad, eficiencia y satisfacción - para ofrecer uno adecuado. Cuatro pacientes con esclerosis lateral amiotrófica evaluaron los tres tamaños. Los participantes tuvieron peor desempeño con el tamaño pequeño, especialmente en la efectividad y satisfacción; mientras que con el mediano obtuvieron mejores resultados, resaltando la satisfacción. Los resultados muestran que se debe considerar el tamaño del teclado al diseñar un nuevo paradigma para personas con discapacidad.
\end{abstract}

Palabras clave: Esclerosis lateral amiotrófica (ELA), Interfaces CerebroComputador (BCI), teclado, P300, tamaño de teclado, usabilidad. 
Estudio preliminar de la usabilidad de tres tamaños de teclados basados en Interfaces CerebroComputador por pacientes con ELA

\section{Introducción}

La Esclerosis Lateral Amiotrófica (ELA) es un desorden neurológico que degenera las neuronas motoras periféricas y centrales, llevando hasta la parálisis total del paciente (Patterson y Grabois, 1986). Sin embargo, se preservan otras funciones como son la percepción sensitiva o las habilidades intelectuales.

Investigadores del campo han desarrollado diferentes sistemas para proporcionar a estos pacientes un canal alternativo de comunicación con su entorno, tales como sistemas eyetracker (Pal, Mangal, y Khosla, 2017), o Interfaces Cerebro Computador (BCI, del inglés Brain-Computer Interfaces) (Birbaumer, 2006). En fases evolucionadas de la enfermedad, los pacientes pueden perder parcial o totalmente el control ocular (imprescindible para controlar sistemas basados en eye-tracker). En estos casos los sistemas BCI sirven como última vía de comunicación para ellos, ya que permiten que los pacientes interactúen con su entorno sin necesidad de realizar movimientos motores, utilizando como señal de control la actividad cerebral del sujeto (Nicolas-Alonso y Gomez-Gil, 2012).

Los sistemas BCI utilizan la electroencefalografía (EEG) para medir la actividad cerebral del sujeto y clasificar las distintas formas de onda como son los ritmos sensoriomotores (SMR, del inglés Sensorimotor Rhythms), o potenciales evocados P300 (P300-EP, del inglés P300 Evoked Potentials).

Este trabajo se centra en los potenciales evocados P300, los cuales son unas variaciones positivas de amplitud de la señal cerebral que aparecen aproximadamente $300 \mathrm{~ms}$ después de que aparezca un estímulo extraño al que se está a la espera. Esta señal es normalmente utilizada por unos BCIs llamados teclados virtuales (Nicolas-Alonso y Gomez-Gil, 2012). Estos sistemas están formados por una matriz de letras, números, símbolos y/o comandos. El paradigma más comúnmente usado es el paradigma oddball propuesto por (Farwell y Donchin, 1988), donde cada columna y fila de la matriz se iluminan de manera pseudoaleatoria. El sujeto debe prestar atención a un elemento específico de la matriz mientras las filas y las columnas son iluminadas, y cuando su elemento objetivo se ilumina, el biopotencial eléctrico P300 es evocado en el cerebro y es reconocido por el sistema.

Anteriormente, se ha estudiado el efecto que producen diversas variaciones visuales de las matrices basadas en P300. Por ejemplo, los colores de los estímulos, la naturaleza de los mismos y el paradigma aplicado (Ikegami et al., 2012; Li et al., 2015; Acqualagna et al., 2013; Ron-Angevin et al., 2015). Las medidas del teclado se han estudiado en escasas ocasiones. En concreto, se ha estudiado el efecto que tienen distintas dimensiones de matrices (Sellers et al., 2006), el efecto que tiene el tamaño de los símbolos (Salvaris y Sepulveda, 2009), el tamaño del monitor en el que se presenta el teclado (Y. Li et al., 2011). Pero ninguno nos da información suficiente para determinar un tamaño óptimo de teclado en cuanto al tamaño de símbolos y distancia entre ellos. Por otra parte, (Ron- 
Angevin et al., 2019) propone en su trabajo un tamaño de teclado óptimo entendido desde el punto de vista de la usabilidad bajo dos condiciones opuestas de movilidad ocular (permitiendo dirigir la mirada al símbolo de interés y manteniendo la mirada en el centro del teclado). El trabajo anterior concluyó que el teclado mediano es el que mejores prestaciones presenta en cuanto al rendimiento (empatado en esta dimensión con el pequeño) y la satisfacción.

Es importante resaltar que todos los artículos arriba mencionados realizaron su estudio con participantes sanos. Por lo que es importante constatar los resultados con los que son realmente los usuarios finales de este tipo de sistema. (Ron-Angevin et al., 2019) estudió una condición que podría ser traducida a pacientes con movilidad ocular reducida (i.e., la condición en la que se impedía mover la mirada del centro del teclado). Sin embargo, esta condición no es del todo realista pues los pacientes podrían conservar movimientos de cara y ojos residuales (Patterson y Grabois, 1986). Además, aprovechar estos movimientos residuales podría ser de gran ayuda para controlar el teclado pues, como (Ron-Angevin et al., 2019) y (Brunner et al., 2010) demostraron, el rendimiento de estos sistemas aumenta significativamente cuando se dirige la mirada al símbolo de interés. Por ello se hace necesario encontrar el tamaño de teclado que, en general, sea usable para los pacientes. Tamaños grandes de teclado podrían encontrarse difícil de manejar no sólo por rango de movimiento ocular limitado sino también por rango de movimiento de cara y cuello limitados. Por otra parte, un teclado muy pequeño podría causar problemas cuando un usuario intente percibir correctamente los diferentes elementos de la matriz, pero podría resultar menos cansado físicamente.

Por tanto, el objetivo del presente trabajo es evaluar de manera preliminar el efecto que provocan en pacientes, diagnosticados con ELA, los tres tamaños de teclados probados por (Ron-Angevin et al., 2019). Los resultados de los participantes fueron analizados individualmente para buscar tendencias relacionas con cualquiera de los teclados. Al igual que (Ron-Angevin et al., 2019), se empleó el modelo de usabilidad según la ISO (2000) para la evaluación; por tanto, tres factores fueron estudiados: efectividad, eficiencia y satisfacción.

\section{Método}

\subsection{Participantes}

Cuatro pacientes españoles diagnosticados con ELA (P1-P4, todos hombres, edad $64.5 \pm$ 6.95) participaron en el presente estudio. Cabe mencionar que el quinto participante con ELA (P5) no pudo participar en el experimento ya que tenía escaso control del parpadeo por lo que tenía problemas para mantener sus ojos abiertos. Todos los participantes (o su correspondiente cuidador) dieron su consentimiento informado. 
Estudio preliminar de la usabilidad de tres tamaños de teclados basados en Interfaces CerebroComputador por pacientes con ELA

Según lo declarado por los participantes, ninguno tenía historial de alguna enfermedad neurológica - además de ELA - o psiquiátrica, y tenían visión normal o corregida. Ninguno de ellos tenía experiencia previa con sistemas BCI. Se contactó con los pacientes a través de la Asociación de ELA de Andalucía. Las pruebas tuvieron lugar en sus respectivas casas. En la Tabla 1 se proporciona información sobre los pacientes. Este estudio fue aprobado por el Comité de Ética de la Universidad de Málaga y cumplió los estándares de ética de la Declaración de Helsinki.

Tabla 1. Información sobre los participantes de este experimento.

\begin{tabular}{ccccc}
\hline Participante & $\begin{array}{c}\text { Edad } \\
\text { (años) }\end{array}$ & $\begin{array}{c}\text { Años post- } \\
\text { diagnóstico }\end{array}$ & ALSFRS-R & $\begin{array}{c}\text { Canal de comunicación } \\
\text { habitual }\end{array}$ \\
\hline P1 & 64 & 4 & 12 & Voz \\
P2 & 73 & 5 & 29 & Manos \\
P3 & 56 & 4 & 0 & Ojos (mirada y pestañeos) \\
P4 & 65 & 20 & 19 & Voz \\
\hline
\end{tabular}

\subsection{Registro y procesado de señales EEG}

Para registrar las señales se utilizó el amplificador Acti-Champ (Brain Products GmbH, Munich, Germany). Las posiciones utilizadas, según el sistema internacional 10/20, fueron Fz, Cz, Pz, Oz, P3, P4, PO7 y PO8, utilizando como referencia la posición TP6 y como tierra AFz. Se controló que la impedancia de la señal se mantuviera $<10 \mathrm{k} \Omega$. La señal se filtró utilizando un paso banda de $0.1-30 \mathrm{~Hz}$. Se utilizó BCI2000 para controlar todos los aspectos de la colección y procesado de los datos.

\subsection{Paradigmas de presentación}

Los tres teclados fueron diseñados acorde a las especificaciones dadas por (Ron-Angevin et al., 2019), donde los tres tamaños tenían una apariencia similar al teclado P300 clásico. Los teclados consistieron en un matriz de caracteres de $6 \times 6$ con el alfabeto inglés y con los números del 0 al 9 (Figura 1). La duración de un flash y el tiempo inter simbólico (ISI) fueron de $128 \mathrm{~ms}$. Después de cada secuencia de flashes había una pausa de $2 \mathrm{~s}$ excepto para el paciente $\mathrm{P} 4$ que utilizó 6s debido a un error en la aplicación del protocolo. En las fases de calibración y escritura libre se utilizaron 10 secuencias. Importante notar que una secuencia consiste en iluminar una vez cada columna y cada fila, por lo que cada carácter es estimulado dos veces por cada secuencia.

Las medidas de los teclados se muestran en la Tabla 2 de acuerdo a los parámetros establecidos en la Figura 1. 


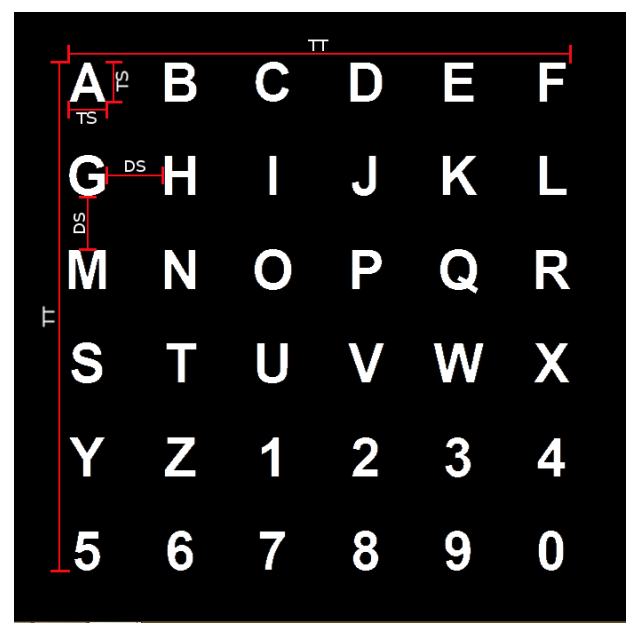

Fig. 1 Tipografia de teclado utilizado. Además, se señalan las medidas consideradas en el presente trabajo. Donde 'TT' simboliza tamaño de teclado, 'TS' tamaño de símbolo y 'DS' distancia entre símbolos.

Tabla 2. Valores de las medidas por tamaño de teclado.

\begin{tabular}{cccc}
\hline \multirow{2}{*}{ Tamaño } & \multicolumn{3}{c}{ Parameter (cm) } \\
\cline { 2 - 4 } & $\begin{array}{c}\text { Tamaño de } \\
\text { teclado (TT) }\end{array}$ & $\begin{array}{c}\text { Tamaño de } \\
\text { símbolo (TS) }\end{array}$ & $\begin{array}{c}\text { Distancia entre } \\
\text { símbolos (DS) }\end{array}$ \\
\hline Pequeño & 5.27 & 0.42 & 0.55 \\
Mediano & 9.98 & 0.79 & 1.04 \\
Grande & 14.69 & 1.17 & 1.53 \\
\hline
\end{tabular}

\subsection{Procedimiento}

En cuanto al procedimiento, se siguió lo indicado por (Ron-Angevin et al., 2019). El experimento consistió en tres sesiones (una por cada teclado). El orden de los teclados fue contrabalanceado para controlar efectos no deseados como la experiencia. En cuanto al tiempo entre sesión y sesión se estableció un rango entre 5 horas y 3 días. En cada sesión se llevaron a cabo las siguientes acciones: i) realizar una tarea de calibración, ii) realizar una tarea de escritura libre y, finalmente, iii) rellenar unos cuestionarios subjetivos referentes al teclado que habían controlado en dicha sesión. En concreto, cada tarea consistió en:

- Tarea de calibración: las palabras a calibrar fueron 'LUNA', 'RAMO', 'KILO' y '2015'. Esta fase tomó aproximadamente 10 minutos.

- Tarea de escritura libre: en esta fase, se pidió a los participantes que deletrearan las palabras 'CHAT', 'PURE' y '1935'. En caso de que el clasificador eligiera una letra errónea, se les indicó que pasaran a la siguiente letra sin posibilidad de corregir el error. 
Estudio preliminar de la usabilidad de tres tamaños de teclados basados en Interfaces CerebroComputador por pacientes con ELA

- Cuestionarios subjetivos: la última parte de cada sesión consistió en contestar dos cuestionario diferentes i) la escala analógica visual (VAS, del inglés visual analogue scale) y ii) el test NASA-TLX. Por último, cuando se completaban las tres sesiones, se debía rellenar un cuestionario comparativo de los tres teclados.

Solo las últimas tres palabras calibradas se utilizaron para la clasificación. El clasificador utilizado fue un Stepwise Linear Discriminant Analysis (SWLDA).

\subsection{Evaluación de la usabilidad}

La evaluación de la usabilidad se llevó a cabo considerando la definición propuesta por ISO (2000), la cual incluye tres dimensiones: efectividad, eficiencia y satisfacción.

\subsubsection{Efectividad}

La efectividad está relacionada con la precisión con la que el usuario completó la tarea. Para esta dimensión se tuvieron en cuenta i) la precisión en la tarea de calibración, y ii) el error de clasificación obtenido en la tarea de escritura libre.

\subsubsection{Eficiencia}

La eficiencia está relacionada con los recursos gastados para completar la tarea. Para evaluarla, se han considerado dos resultados: i) la carga de trabajo subjetiva utilizando el NASA-TLX, ii) el cuestionario VAS sobre preguntas relacionadas con características de los teclados. Cada uno de éstos evalúa los siguientes parámetros:

- $\quad$ NASA-TLX: evaluó la demanda mental, física y temporal, así como el desempeño, el esfuerzo y la frustración percibida por el participante. La predominancia de cada uno de estos parámetros en la carga de trabajo total es computada en escala del 0-100 como media de pesos, donde la puntuación máxima de cada parámetro es 33.33.

- VAS: se ponderan cada uno de los parámetros de 0-10 (donde 0 es el mínimo y 10 el máximo). Lo que evaluó fue: i) la dificultad en percibir los caracteres alejados del centro, y ii) la dificultad en distinguir las diferentes columnas y filas.

\subsubsection{Satisfacción}

Finalmente, la satisfacción está relacionada con la actitud de los usuarios. La preferencia y lo sentido por el usuario fue analizado utilizando un cuestionario comparativo basado en la Escala de la Usabilidad del Sistema (SUS) (Brooke, 1996). En este cuestionario se les pidió 
a los usuarios que puntuaran por cada teclado el nivel de complejidad, estrés causado, sensación de control, cansancio, y preferencia.

\section{Resultados y discusión}

En esta sección los resultados de cada paciente son presentados individualmente ya que no fue posible realizar un estudio estadístico por el bajo tamaño muestral. Al mismo tiempo que se presentan los resultados se irán discutiendo y comparando con la literatura.

\subsection{Efectividad}

En este apartado se muestran los resultados en apartados distintos según la precisión alcanzada en la fase de calibración y el error obtenido en la fase de escritura libre.

\subsubsection{Precisión obtenida en la fase de calibración}

Se ha realizado un análisis estadístico de caso único para cada uno de los participantes. La precisión alcanzada por cada participante se puede observar en la Figura 2.

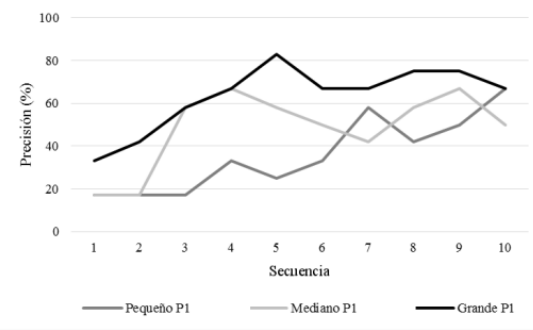

a)

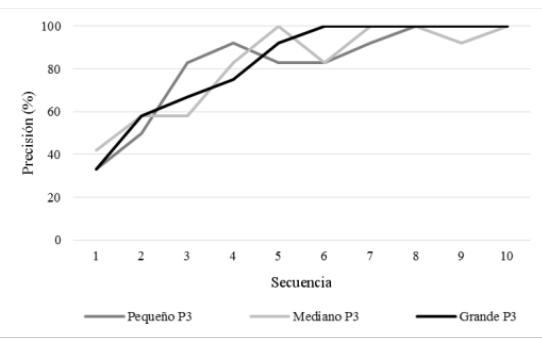

c)

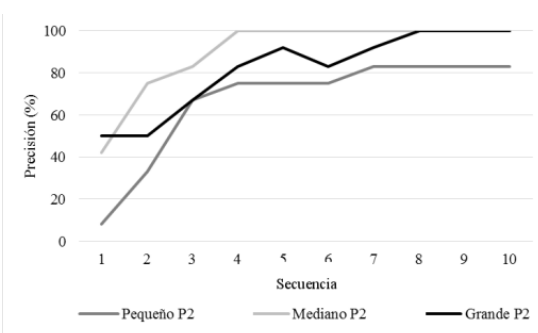

b)

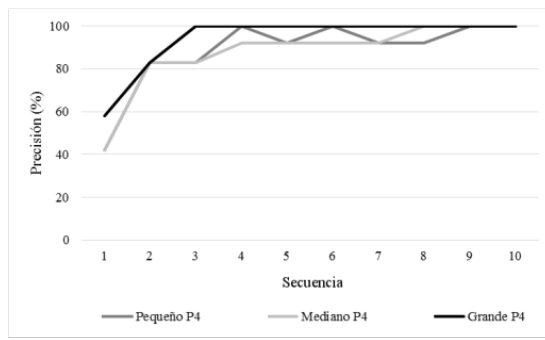

d)

Fig. 2 Resultados de la fase de calibración de los cuatro participantes al utilizar los tres tamaños de teclado: a) corresponde a los resultados del participante P1, b) a los del participante P2, c) a los del P3, yd) a los del P4. 
Estudio preliminar de la usabilidad de tres tamaños de teclados basados en Interfaces CerebroComputador por pacientes con ELA

A continuación se detallan los resultados de cada uno de los participantes:

- Participante P1: este participante obtuvo una precisión relativamente baja durante esta fase con los tres tamaños: pequeño, $35.9 \pm 17.95 \%$; mediano, $48.4 \pm 18.22 \%$; y grande, $63.4 \pm 15.33 \%$. Se obtuvieron diferencias significativas con respecto al teclado utilizado $(F(2,18)=14.573 ; p<.001)$. En concreto, el teclado grande obtuvo una precisión significativamente mejor que la de los teclados mediano y pequeño ( $p=.002$, en ambos casos). Aunque no se obtuvieron diferencias significativas entre el teclado pequeño y mediano, mirando los resultados obtuvo peor desempeño con el teclado pequeño (Figura 2.a).

- Participante P2: sus resultados fueron: pequeño $66.5 \pm 25.5 \%$, mediano $90 \pm 19.1 \%$ y grande $81.7 \pm 19.57 \%$. Se obtuvieron diferencias significativas con respecto al teclado $(F(2,18)=27.847 ; p<.001)$. En concreto, el teclado pequeño obtuvo significativamente peor rendimiento que los teclados grande $(p=.006)$ y mediano $(p$ $<.001)$. Aunque no se obtuvieron resultados significativamente mejores, viendo la Figura 2.b, se observa que tuvo mejor rendimiento con el mediano.

- Participante P3: obtuvo como resultados: pequeño $81.6 \pm 22.6 \%$, mediano $81.6 \pm$ $21.47 \%$ y grande $82.5 \pm 23.5 \%$. En este caso no se obtuvieron diferencias significativas con respecto al tamaño de teclado utilizado. Mirando a la Figura 2.c, sus mejores resultados los obtuvo con los teclados mediano y grande.

- Participante P4: este sujeto fue el participante que consiguió los mejores resultados: para el tamaño pequeño $88.4 \pm 17.6 \%$, para el mediano $87.6 \pm 17.19 \%$ y el grande $94.1 \pm 13.76 \%$. Se obtuvieron diferencias significativas entre teclados $(F(2,18)=$ $6.95, p=.006)$. En concreto, la precisión del teclado grande fue significativamente mejor que la del teclado mediano $(p=.034)$.

Ninguno de los teclados puede ser considerado como el mejor, sin embargo, parece que el teclado grande ofrece mejores resultados por lo menos para dos de los participantes. De igual forma, se puede observar que el teclado pequeño es el que en general ha obtenido los peores resultados, dejando al mediano entre ambos teclados. Al existir diferencias individuales, se enfatiza la importancia de que el tamaño debe adaptarse a las características y preferencias del sujeto.

Observando el resultado obtenido en la décima secuencia, la mayoría los participantes consiguieron 100\%; en concreto, P3 y P4 con los tres teclados, y P2 con el grande y el mediano. En el caso de P1, no consiguió 100\% con ninguno de ellos. Esta información es importante pues esta secuencia es la que se eligió como parámetro fijo para la fase online. Cabe resaltar que P2 y P3 consiguieron su precisión más alta con menos número de secuencias con el teclado mediano (en secuencia 4 y 5 , respectivamente), mientras que P1 y P4 con el teclado grande (en las secuencias 4 y 5 , respectivamente) considerando que P1 no alcanzó $100 \%$. Por lo tanto, para todos los sujetos, el teclado pequeño es el menos 
recomendado. Comparando el resultados de estos sujetos con los obtenidos por (RonAngevin et al., 2019), tanto los participantes de este experimento como los sujetos sanos consiguieron sus puntuaciones máximas en el mismo rango de secuencias (i.e., entre 4 y 6 ). Estos resultados también concuerdan con los obtenidos por (Ryan et al., 2018) por otros participantes con ELA que controlaron una matriz de $6 \times 6$, pues obtuvo que los participantes necesitaron entre 4 y 7 secuencias para alcanzar su puntuación máxima.

\subsubsection{Error obtenido en la fase de escritura libre}

La Tabla 3 muestra el error obtenido en la fase de escritura libre para los cuatro participantes. El error se calculó dividendo el número de errores entre los 12 caracteres a deletrear.

Tabla 3. Porcentaje de error obtenido en la tarea de escritura libre. El error se calculó dividiendo el número de errores por el número total de caracteres deletreados.

\begin{tabular}{cccc}
\hline \multirow{2}{*}{ Participante } & \multicolumn{3}{c}{ Tamaño de teclado } \\
\cline { 2 - 4 } & Pequeño & Mediano & Grande \\
\hline P1 & 83.33 & 41.67 & 58.33 \\
P2 & 33.33 & 25 & 25 \\
P3 & 0 & 0 & 16.67 \\
P4 & 8.33 & 8.33 & 0 \\
\hline
\end{tabular}

A continuación, se detallan los resultados obtenidos por cada uno de los participantes:

- Participante P1: para P1 el mejor teclado fue el mediano, consiguiendo un error de $41.667 \%$ y el peor el pequeño con un error del $83.33 \%$.

- Participante P2: este participante obtuvo el mismo resultado con los teclados grande y mediano (25\% de error), y con el pequeño obtuvo su peor resultado $(33.33 \%)$.

- Participante P3: este participante obtuvo el mismo porcentaje de error con el teclado pequeño y mediano ( $0 \%$ ), mientras que con el grande obtuvo un $16.67 \%$ (lo que significa que sólo cometió dos errores).

- Participante P4. Este participante obtuvo su mejor resultado con el teclado grande $(0 \%)$. Con los otros dos obtuvo el mismo porcentaje de $8.33 \%$, lo que corresponde a un único error.

Comparando los resultados de los cuatro participantes con otros estudios con pacientes con ELA que han utilizado teclados basados en el de (Farwell y Donchin, 1988) y tamaños de matriz grande (ej., (McCane et al., 2014; Nijboer et al., 2008; Poletti et al., 2016)), se pude observar que los resultados obtenidos son representativos de la población. 
Estudio preliminar de la usabilidad de tres tamaños de teclados basados en Interfaces CerebroComputador por pacientes con ELA

El teclado mediano ofreció los mejores resultados en términos de error para P1 (46.67\%), P2 (25\%, junto con el tamaño grande) y P3 ( $0 \%$, junto con el pequeño). Por el contrario, el pequeño fue el que obtuvo los peores resultados para P1 (83.33\%), P2 (33.33\%) y P4 (8.33\%, junto con el tamaño mediano). Comparando los resultados de los pacientes entre ellos, P1 y P2 fueron los que peores resultados obtuvieron. Esto pudo ser debido a los $2 \mathrm{~s}$ de pausa entre letras configurado, ya que este tiempo podría no haber sido suficiente para ellos para dirigir la mirada al siguiente símbolo, lo cual pudo haber afectado su desempeño. Sin embargo, esta pausa se mantuvo en los tres teclados, por lo que esto podría no ser relevante para el estudio.

Por otra parte, si utilizamos el criterio de (Kubler et al., 2001) el cual indica que $30 \%$ es el error máximo permitido para establecer un sistema de comunicación eficiente, el teclado pequeño es usable solo por el 50\% de los participantes (P3 y P4), mientras que el mediano y grande para el 75\% de ellos (P2, P3 y P4 en ambos casos). Estos resultados indican que el pequeño puede ser el que aporte menos beneficios.

\subsection{Eficiencia}

En esta sección se muestran los resultados obtenidos en los cuestionarios subjetivos, donde cada uno de ellos (i.e., NASA-TLX y VAS) corresponden a un apartado distinto.

\subsubsection{NASA-TLX}

En la Figura 3 se muestran las puntuaciones ponderadas de los parámetros por sujeto.

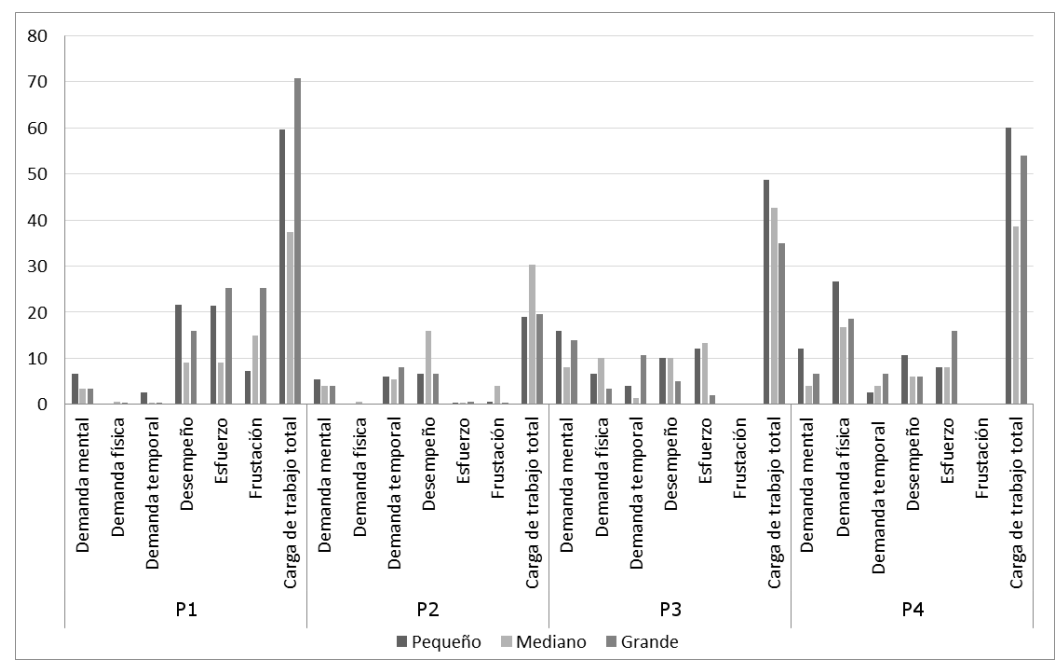

Fig. 3 Resultados obtenidos en el test NASA-TLX con sus parámetros ya ponderados. 
A continuación, se detallan los resultados por participante:

- Participante P1: puntuó con los valores más bajos al teclado mediano (carga de trabajo total, 37.33). Estas diferencias fueron sobretodo observables en el desempeño (puntuación ponderada, 9), esfuerzo (puntuación ponderada, 9), y frustración (puntuación ponderada, 15). Por otra parte, al teclado que puntuó con valores más altos fue al teclado grande (carga de trabajo, 70.67), especialmente remarcable en las dimensiones esfuerzo y frustración (ambas puntuadas ponderadamente con 25.33).

- Participante P2: este sujeto puntuó con los valores más altos al teclado mediano (carga de trabajo total, 30.33), mientras que a los otros dos los puntuó con valores más bajos muy cercanos (19 y 19.67 al pequeño y al grande, respectivamente). El mediano obtuvo principalmente una puntuación relativamente alta en la dimensión desempeño (puntuación ponderada, 16).

- Participante P3: es difícil encontrar tendencias claras con los resultados de este participante. Sin embargo, parece que la carga de trabajo aumenta conforme disminuye el tamaño del teclado.

- Participante P4. Con este participante la tendencia se ve clara. El teclado mediano obtuvo los valores más bajos (carga de trabajo total, 38.67), sobretodo en las variables demanda mental (puntuación ponderada, 4), física (puntuación ponderada, 16.67) y temporal (puntuación ponderada, 8). El tamaño que obtuvo las mayores puntuaciones fue el pequeño (carga de trabajo total, 60), resaltando la demanda mental y fisica (puntuación ponderada, 12 y 26.67, respectivamente) y el desempeño (puntuación ponderada, 10.68).

El trabajo realizado por (Pasqualotto et al., 2015), consiste en un estudio de la usabilidad de los teclados BCI con personas con ELA. En dicho estudio se obtuvo una carga total media de $47.64 \pm 14.87$, la cual engloba a los resultados obtenidos para el teclado grande de tres de los participantes del presente estudio, excepto P1, el cual declaró una carga de trabajo muy por encima de la media (70.67). En (Ron-Angevin et al., 2019), las medias de carga de trabajo para cada uno de los tamaños (pequeño: $40.4 \pm 7.2$, mediano: $38.22 \pm 4.8$, grande: $41.2 \pm 6.4$ ) engloban los resultados de los tres teclados de P3 y los de los teclados medianos de $\mathrm{P} 1$ y $\mathrm{P} 4$, mientras que son menores con respecto a los resultados de los teclados grande y pequeño de P1 y P4, y mayores con respecto a los tres de P2.

\subsection{2. $V A S$}

Esta sección presenta los resultados obtenidos en el cuestionario VAS sobre la percepción subjetiva (véase la Tabla 4 para ver los resultados de los participantes). 
Estudio preliminar de la usabilidad de tres tamaños de teclados basados en Interfaces CerebroComputador por pacientes con ELA

Tabla 4. Respuestas al cuestionario VAS.

\begin{tabular}{ccccccc}
\hline \multirow{2}{*}{ Participante } & \multicolumn{5}{c}{ Pregunta sobre percepción para cada tamaño } \\
\cline { 2 - 7 } & \multicolumn{4}{c}{ Q1 } & Q2 \\
\cline { 2 - 7 } & $\mathrm{P}$ & $\mathrm{M}$ & $\mathrm{G}$ & $\mathrm{P}$ & $\mathrm{M}$ & $\mathrm{G}$ \\
\hline P1 & 2 & 2 & 6 & 5 & 2 & 2 \\
P2 & 0 & 0 & 0 & 0 & 0 & 0 \\
P3 & 0 & 0 & 0 & 0 & 0 & 0 \\
P4 & 8 & 5 & 7 & 6 & 5 & 2 \\
\hline
\end{tabular}

Nota: Q1 simboliza la 'Dificultad para percibir los simbolos alejados del centro'; Q2 la 'Dificultad para distinguir entre las filas y las columnas'; $P$ representa 'pequeño', $M$ 'mediano' y $G$ 'grande'. La puntuación varía de 0 a $10(0=$ muy fácil; $10=$ muy dificil).

Por una parte, P1 y P3 declararon no haber tenido alguna dificultad en cuanto a las preguntas planteadas sobre los teclados. Nosotros pensamos que es debido a la actitud positiva y a la gran motivación que presentaron dichos participantes. Por otra parte, P1 y P4 coinciden en que el tamaño pequeño es el más complicado para distinguir entre las distintas filas y columnas $(Q 2)$. Mientras que la primera pregunta $(Q 1)$ está contrabalanceada en los otros teclados. Dados los resultados, parece complicado destacar tendencias de ellos.

Es interesante resaltar que $\mathrm{P} 4$ ha seleccionado puntuaciones parecidas a las obtenidas por los participantes sanos de (Ron-Angevin et al., 2019) (Q1: pequeño: $2.2 \pm 0.7$, mediano: 1.2 \pm 0.4 , grande: $1.8 \pm 0.5$; Q2: pequeño: $2.7 \pm 0.8$, mediano: $1.15 \pm 0.4$, grande: $1.6 \pm 0.5$ ) Puesto que este participante alcanzó puntuaciones parecidas o incluso mejores en la parte de eficiencia que la media de los participantes de dicho estudio. En cuanto a P1, la puntuación de $Q 1$ puede estar relacionada con la carga de trabajo total pues en ambos casos puntuó negativamente al teclado grande. Pensamos que ambas puntuaciones pueden estar relacionadas con su declarada dificultad para percibir todas las letras. Sin embargo, con el teclado grande y mediano fue con los que obtuvo mejores resultados en la parte de la eficiencia, lo cual puede ser debido a una mayor facilidad para distinguir las filas y las columnas (i.e., $Q 2)$.

\subsection{Satisfacción}

En la Figura 4 se puede observar en forma de porcentaje los participantes que votaron a uno u otro teclado según el parámetro y ranking. A pesar de no poder realizar análisis estadístico debido al bajo tamaño muestral, parece claro que el teclado mediano es el que ha obtenido los mejores resultados en todas las variables: fue encontrado el menos complejo (seleccionado por 3 de 4), estresante (seleccionado por los 4), y cansado (seleccionado por 4 de 4), además del más controlable, preferido (seleccionado por 4 de 4 , en ambas variables) y cómodo (seleccionado por 3 de 4). Por otra parte, el teclado pequeño se 
seleccionó como el más complejo (seleccionado por 3 de 4), más estresante (seleccionado por 4 de 4), el menos cómodo (seleccionado por 3 de 4) y menos controlable (seleccionado por 3 de 4).

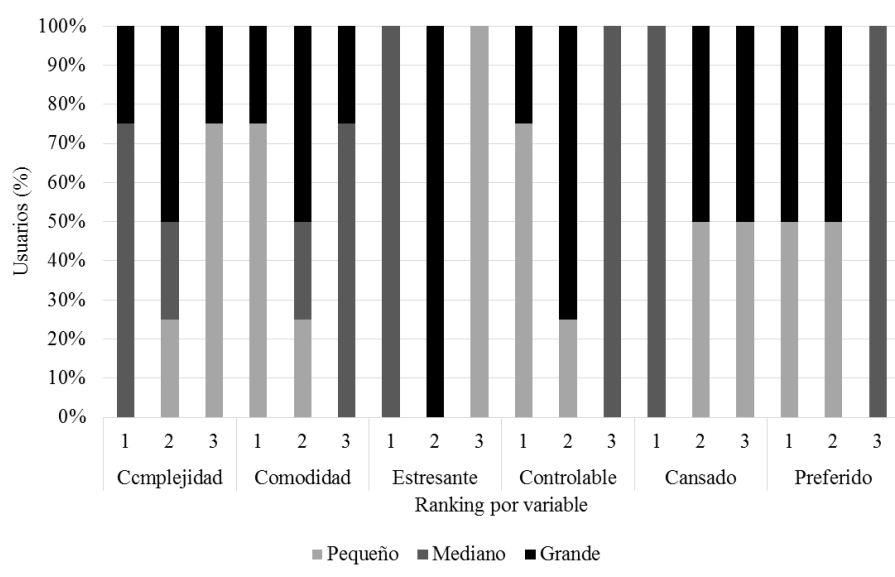

Fig. 4 Resultados obtenidos en el test comparativo. En las barras se presenta el porcentaje de participantes que eligieron un teclado u otro en cada una de los rankings. El Ranking 1 representa 'el menos', el Ranking 2 'el intermedio' y el Ranking 3 'el más'.

La preferencia casi unánime en todas las variables con respecto al teclado mediano, puede ser explicada en parte por la dificultad de los pacientes en fijar su atención en aquellos símbolos alejados del centro (i.e., Q1) con respecto al teclado grande. Por otra parte, la no preferencia del teclado pequeño puede ser explicada por la dificultad en percibir las distintas filas y columnas (i.e., $Q 2$ ).

\section{Conclusiones}

Primero, es importante resaltar que todas las conclusiones no son significativas y que deben ser tomas con cautela. Por otra parte, gracias a los resultados obtenidos en este estudio preliminar, se concluye que el tamaño del teclado sí es relevante para aquellas personas con movilidad reducida. Además, esto también indica que el tamaño de teclado más comúnmente utilizado (i.e., el teclado de tamaño grande) puede no ser el más adecuado a utilizar.

(Ron-Angevin et al., 2019) concluyó que para los sujetos sanos, el mejor tamaño de teclado es el mediano, lo cual lo corroboran las tres dimensiones de la propuesta de usabilidad. Sin embargo, en este caso, cuesta trabajo diferenciar el tamaño óptimo de los resultados de las tres dimensiones. De la parte de eficacia destacan tanto el grande como el mediano, y de la satisfacción se ve la tendencia clara del mediano. Por otra parte, de los tres teclados 
Estudio preliminar de la usabilidad de tres tamaños de teclados basados en Interfaces CerebroComputador por pacientes con ELA

utilizados, solo el teclado pequeño es el que se puede definir como el menos usable viendo el desempeño y puntuaciones obtenidas por los cuatro participantes en las fases de eficacia y satisfacción.

\section{Agradecimientos}

Este trabajo fue parcialmente financiado por el Ministerio Español de Economía y Competitividad a través del proyecto LICOM (DPI2015-67064-R), el Fondo Europeo de Desarrollo Regional (FEDR) y la Universidad de Málaga. Los autores quieren agradecer especialmente a la Fundación Andaluza de ELA y a los participantes por su entusiasmo y cooperación.

\section{Referencias}

ACQUALAGNA, L., TREDER, M. S., \& BLANKERTZ, B. (2013). Chroma Speller: Isotropic visual stimuli for truly gaze-independent spelling. International IEEE/EMBS Conference on Neural Engineering, NER, 1041-1044. https://doi.org/10.1109/NER.2013.6696115

BIRBAUMER, N. (2006). Breaking the silence: Brain-computer interfaces (BCI) for communication and motor control. Psychophysiology, 43(6), 517-532. https://doi.org/10.1111/j.14698986.2006.00456.x

BROOKE, J. (1996). SUS - A quick and dirty usability scale. Usability Evaluation in Industry, 189(194), 4-7. https://doi.org/10.1002/hbm.20701

BRUNNER, P., JOSHI, S., BRISKIN, S., WOLPAW, J. R., BISCHOF, H., \& SCHALK, G. (2010). Does the "P300" speller depend on eye gaze? Journal of Neural Engineering, 7(5). https://doi.org/10.1088/1741-2560/7/5/056013

FARWELL, L. A., \& DONCHIN, E. (1988). Talking off the top of your head: toward a mental prosthesis utilizing event-related brain potentials. Electroencephalography and Clinical Neurophysiology, 70(6), 510-523. https://doi.org/10.1016/0013-4694(88)90149-6

IKEGAMI, S., TAKANO, K., WADA, M., SAEKI, N., \& KANSAKU, K. (2012). Effect of the green/blue flicker matrix for P300-based brain-computer interface: An EEG-fMRI study. Frontiers in Neurology, JUL(July), 1-10. https://doi.org/10.3389/fneur.2012.00113

ISO. (2000). ISO/DIS 9241-9, Ergonomic requirements for office work with visual display terminals. Technical Report, 11, 22. https://doi.org/citeulike-article-id:3503574

KUBlER, A., NEUMANN, N., KAISER, J., KOTCHOUBEY, B., HINTERBERGER, T., \& BIRBAUMER, N. P. (2001). Brain-computer communication: Self-regulation of slow cortical potentials for verbal communication. Archives of Physical Medicine and Rehabilitation, 82(11), 1533-1539. https://doi.org/10.1053/apmr.2001.26621

LI, Q., LIU, S., LI, J., \& BAI, O. (2015). Use of a green familiar faces paradigm improves P300speller brain-computer interface performance. PLOS ONE, 10(6), 1-15. https://doi.org/10.1371/journal.pone.0130325

LI, Y., NAM, C. S., SHADDEN, B. B., \& JOHNSON, S. L. (2011). A p300-based brain-computer 
interface: Effects of interface type and screen size. International Journal of Human-Computer Interaction, 27(1), 52-68. https://doi.org/10.1080/10447318.2011.535753

MCCANE, L. M., SELlERS, E. W., MCFARLAND, D. J., MAK, J. N., CARMACK, C. S., ZEITLIN, D., ... VAUGHAN, T. M. (2014). Brain-computer interface (BCI) evaluation in people with amyotrophic lateral sclerosis. Amyotrophic Lateral Sclerosis and Frontotemporal Degeneration, 15(3-4), 207-215. https://doi.org/10.3109/21678421.2013.865750

NICOLAS-ALONSO, L. F., \& GOMEZ-GIL, J. (2012). Brain computer interfaces, a review. Sensors, 12(2), 1211-1279. https://doi.org/10.3390/s120201211

NIJBOER, F., SELlERS, E. W., MELLINGER, J., JORDAN, M. A., MATUZ, T., FURDEA, A., ... KÜBLER, A. (2008). A P300-based brain-computer interface for people with amyotrophic lateral sclerosis. Clinical Neurophysiology, 119(8), 1909-1916. https://doi.org/10.1016/j.clinph.2008.03.034

PAL, S., MANGAL, N. K., \& KHOSLA, A. (2017). Development of assistive application for patients with communication disability. IEEE International Conference on Innovations in Green Energy and Healthcare Technologies - 2017, IGEHT 2017, 1-4. https://doi.org/10.1109/IGEHT.2017.8094044

PASQUAlOtTO, E., MATUZ, T., FEDERICI, S., RUF, C. A., BARTL, M., OLIVETTI BELARDINELLI, M., ... HALDER, S. (2015). Usability and Workload of Access Technology for People with Severe Motor Impairment: A Comparison of Brain-Computer Interfacing and Eye Tracking. Neurorehabilitation and Neural Repair, 29(10), 950-957. https://doi.org/10.1177/1545968315575611

PATTERSON, J. R., \& GRABOIS, M. (1986). Locked-in syndrome: A review of 139 cases. Stroke, 17(4), 758-764. https://doi.org/10.1161/01.STR.17.4.758

POLETTI, B., CARELli, L., SOlCA, F., LAFRONZA, A., PEDROLI, E., FAINI, A., ... SILANI, V. (2016). Cognitive assessment in Amyotrophic Lateral Sclerosis by means of P300-Brain Computer Interface: a preliminary study. Amyotrophic Lateral Sclerosis and Frontotemporal Degeneration, 17(7-8), 473-481. https://doi.org/10.1080/21678421.2016.1181182

RON-ANGEVIN, R., VARONA-MOYA, S., \& SILVA-SAUER, L. DA. (2015). Initial test of a T9like P300-based speller by an ALS patient. Journal of Neural Engineering, 12(4). https://doi.org/10.1088/1741-2560/12/4/046023

RON-ANGEVIN, R., GARCIA, L., FERNÁNDEZ-RODRIGUEZ, Á, SARACCO, J., ANDRÉ, J.M., \& LESPINET-NAJIB, V. (2019) Impact of speller size in a visual P300-brain-computer interface (BCI) system under two conditions of constraint for eyes movement. Computational Intelligence and Neuroscience, Accepted Manuscript.

RYAN, D. B., COLWELL, K. A., THROCKMORTON, C. S., COLlinS, L. M., CAVES, K., \& SELLERS, E. W. (2018). Evaluating Brain-Computer Interface Performance in an ALS Population: Checkerboard and Color Paradigms. Clinical EEG and Neuroscience, 49(2), 114121. https://doi.org/10.1177/1550059417737443

SALVARIS, M., \& SEPULVEDA, F. (2009). Visual modifications on the P300 speller BCI paradigm. Journal of Neural Engineering, 6(4). https://doi.org/10.1088/1741-2560/6/4/046011

SELLERS, E. W., KRUSIENSKI, D. J., MCFARLAND, D. J., VAUGHAN, T. M., \& WOLPAW, J. R. (2006). A P300 event-related potential brain-computer interface (BCI): The effects of matrix size and inter stimulus interval on performance. Biological Psychology, 73(3), 242-252. https://doi.org/10.1016/j.biopsycho.2006.04.007 